\title{
Recent advances in application of amino acids: Key building blocks in design and syntheses of heterocyclic compounds
}

\author{
Majid M. Heravi ${ }^{1}$, VaezehFathi Vavsari \\ ${ }^{1}$ Department of Chemistry, Faculty of Physics \& Chemistry Alzahra University, Vanak, Tehran, Iran.
}

\begin{abstract}
Amino acids (AAs) are usually considered as important scaffolds for peptides and proteins. However, in the past decades, they have been extensively used as important synthons for the syntheses of a variety of heterocyclic systems, especially for those in which one particular stereoisomer should be obtained via asymmetric synthesis. Although the applications of AAs as synthons in heterocyclization were extensively reviewed in 1995, numerous recent reports concerning their use for the construction of a wide variety of differently sized heterocycles make the subject worthwhile of update.
\end{abstract}

\section{Keywords}

Amino acids, Natural products, Asymmetric synthesis, Bifunctional, Chiral catalyst, Heterocyclization, 